\title{
Adaptive Fixed-time Control for Nonlinear Systems Against Time-varying Actuator Faults
}

\author{
Yu Mei \\ Anhui University of Technology \\ Jing Wang \\ Anhui University of Technology \\ Ju H. Park \\ Yeungnam University \\ Kaibo Shi \\ Chengdu University \\ Hao Shen ( $\square$ haoshen10@gmail.com ) \\ Anhui University of Technology https://orcid.org/0000-0001-7024-6573
}

\section{Research Article}

Keywords: Uncertain nonlinear systems, backstepping method, unknown actuator faults, adaptive fixedtime control

Posted Date: November 2nd, 2021

DOI: https://doi.org/10.21203/rs.3.rs-1023045/v1

License: (c) (i) This work is licensed under a Creative Commons Attribution 4.0 International License. Read Full License

Version of Record: A version of this preprint was published at Nonlinear Dynamics on January 6th, 2022. See the published version at https://doi.org/10.1007/s11071-021-07171-y. 


\title{
Adaptive fixed-time control for nonlinear systems against time-varying actuator faults
}

\author{
Yu Mei · Jing Wang • Ju H. Park (区) · Kaibo Shi • \\ Hao Shen (四)
}

Received: date / Accepted: date

\begin{abstract}
The adaptive fixed-time control problem for nonlinear systems with time-varying actuator faults is investigated in this paper. A novel adaptive fixed-time controller is designed via combining the Lyapunov stability theory with the backstepping method. It can be adapted to both system uncertainties and unknown actuator faults. Compared with the existing fault-tolerant control schemes subject to actuator faults, the adaptive fixed-time neural networks control scheme can make sure that the tracking error is convergent in a small neighborhood of the origin within a fixed-time interval, and it does not depend on the original states of the system and actuator faults. In light of the control scheme proposed in this paper, the fixed-time stability of the closed-loop system can be guaranteed by theoretical analysis, and a numerical example is provided to verify the effectiveness of obtained theoretical results.
\end{abstract}

Keywords Uncertain nonlinear systems · backstepping method · unknown actuator faults · adaptive fixed-time control

\section{Introduction}

In most practical control systems, sensor, actuator and the plant itself faults may occur at uncertain time, which can lead to poor performance and even instability of control systems. Therefore, it is significant to improve system reliability and safety not only by designing fault tolerant control (FTC) to compensate the effect caused by faults automatically, but also by enhancing reliability of signal components. The approaches of the fault-tolerant design can be broadly divided into two categories: the passive one [1-5] and the active one [6-11]. Although the passive approach is usually exploited to handle partial actuator faults [12-14] and complete actuator faults [15-17], it has also a limited capability of handling unknown actuator faults due to its passive control laws being fixed. Unlike passive approaches, active ones consist in reconstructing the controller online, which are more capable of coping with unknown actuator faults. Based on existing results, a mass of active approaches are designed online by employing different approaches, such as adaptive method [18], sliding mode control method [19] and fuzzy control method $[20]$.

In particular, among active methods, it seems that an adaptive control method is a most representative solution. For example, an adaptive FTC was presented for unknown uncertain systems (UNSs) in [21] where the fault-tolerant capacity was enhanced by applying adaptive bounding design techniques. However, in [21], the system uncertainty needs to meet the matching conditions, which is not easy to achieve in practical system. Therefore, based on the demand of the constraint of the system uncertainty meeting the matching condition being released, the backstepping method is introduced into adaptive FTC. Since

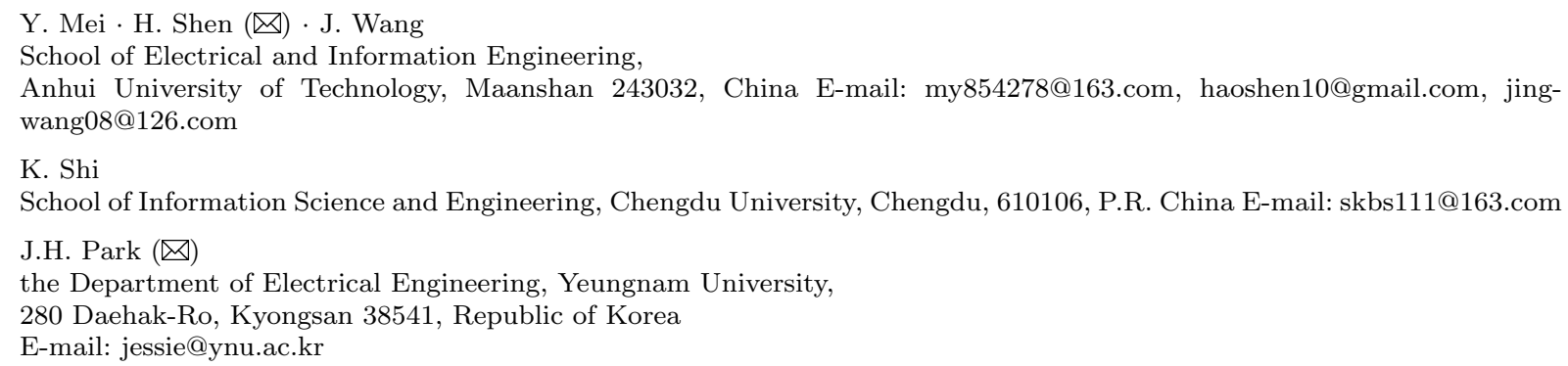


backstepping approaches were proposed, they were been widely used to design adaptive FTC for UNSs [22-26]. For instance, in [27] and [28], based on the backstepping technique, adaptive FTC approaches were designed to handle actuator faults in nonlinear systems where the FTC method runs through the entire backstepping control when there exist actuator faults and guarantee tracking precision. Identically, in [29], the combination of backstepping approaches and adaptive FTC with UNSs greatly also improves the system stability and accuracy. However, the analysis of FTC is usually at infinite time, namely, FTC is able to ensure the system performance of the origin when time tends to infinity. Obviously, during a highly critical mission, such the infinite time may not be an optimal option. Actually, in finite time [30-34], that the tracking error is convergent is always required even when faults occur. After learning sufficient literature, finite-time Lyapunov stability was first presented in [35]. Up to now, abundant results have been obtained under the circumstance of nonlinear systems [36-38]. Based on FTC stability, [39] and [40] obtained some interesting results in regard to finite-time FTC with actuator faults. Specifically, the finite-time FTC for UNSs against actuator faults was investigated in [39] by neural networks (NNs) and fuzzy logic systems. It is worth noting that most results which include actuator faults only involved bias faults, not gain faults. In practical operation, the abrupt faults which cover bias faults and gain faults are very difficult to avoid. With the development of modern control theory, [40] was devoted to handling the finite-time FTC problem of UNSs with such faults. In addition, adaptive finite-time fuzzy tracking control with bias and gain faults was considered in [41-43] for UNSs. Although there exist a mass of literatures on tracking control, there is a restriction in the existing literature on finite-time tracking control of UNSs, that is, the designed finite-time control laws might not satisfy the demand of some applications because of dependent on the initial conditions.

Due to the harsh external environment, the initial conditions of the system may not be known. To deal with the unknown initial conditions of finite-time control problems, the fixed-time stability was proposed by Polyakov in [44], in which it estimated the settling time, and it is independent on the initial conditions. Recently, a mass of research results on the fixed-time have been achieved for first-order dynamic systems [45], second-order dynamic systems [46]. However, there exists little literature for higher-order dynamic systems. Inspired by this, a high-order dynamic non-strict feedback system is adopted in this paper. However, due to existing uncertainties, a traditional form of fixed time stability convergence is hard to obtain. To solve this dilemma, lots of adaptive FTC are introduced into existing research by using radial bais function neural networks (RBFNNs) [47]. However, in the above results of FTC, most schemes focus on the finite-time stable. In [48], within a finite time interval, a class of UNSs is able to track the reference signal suffering from both bias faults and gain faults. The gain which has time-varying form and uncertain bias faults are solved by introducing the virtual error parameter. Nevertheless, convergence time of [48] depends on the initial conditions, which might not meet some applications demand. Therefore, how to obtain adaptive fixed-time FTC becomes a challenging issue.

Motivated by the above observations, a solution to cope with the fixed-time tracking issue for nonlinear systems subject to actuator faults is proposed in this work, in which the RBFNNs control strategy is to approximate unknown functions where both time-varying and bias fault are considered. The main contributions proposed to achieve them are listed as follows.

1) The time-varying gain fault and the unknown bias fault are considered in this paper, whose purpose is handling faults to receive the fixed-time stability, which is the major difficulty to be addressed. The problem of time-varying gain is solved by introducing virtual parameters, and the unknown bias fault is handled, which is assumed to be bounded. Accordingly, the proposed scheme can guarantee that the output tracks the reference signal as quickly as possible with a fixed time.

2) By introducing RBFNNs into the backstepping design, the uncertainty of the nonlinear term is well resolved. Compared with fuzzy logic systems, the RBFNNs not only have the property of easy design and better generalization, but also can linearize the parameters. In addition, it only needs to update online the output layer and a new designed controller can cope with the interactions among variables and coupling terms effectively, which reduces the computational complexity.

3) The newly proposed fixed-time controller has an advantage over the finite-time tracking control where it can guarantee a higher tracking precision. Moreover, the newly proposed controller is more excellent than finite-time results and the settling time of our newly proposed controller which is independent of initial conditions can be designed in advance by modifying parameters.

This article is organized as follows. Section 2 gives the system description and basic assumption. In Section 3, the novel adaptive fixed-time scheme is proposed. Section 4 provides a simulation example to show the effectiveness of the designed scheme. Finally, Section 5 contains conclusions and prospects for the future. 


\section{Problem Statement}

\subsection{Basic Assumption and System Description}

An uncertain nonlinear system with actuator faults in this paper is considered as the following form

$$
\left\{\begin{array}{l}
\dot{x}_{j}=x_{j+1}+f_{j}\left(\bar{x}_{j}\right), 1 \leq j \leq m-1 \\
\dot{x}_{m}=u^{F}+f_{m}\left(\bar{x}_{m}\right) \\
y=x_{1}
\end{array}\right.
$$

where $\bar{x}_{j}=\left[x_{1}, \ldots, x_{j}\right]^{T} \in R^{j}$ denote the system states; $u^{F}$ is the input of the system; $y$ is the output of the system; $f_{j}\left(\bar{x}_{j}\right)$ denotes an smooth unknown nonlinear function; for such an uncertain nonlinear system, $u_{k}$ is designed when the system is normal. However, in the actual system, there exist failures in the actuators. The following actuator fault model borrowed from [49] and [50] is adopted in this paper

$$
u^{F}=b\left(t, t_{b}\right) u_{k}+u_{\sigma}\left(t, t_{\sigma}\right)
$$

in which the actuator fault $b\left(t, t_{b}\right) \in[0,1]$ under consideration is the unknown time-varying fault; $u_{k}$ represents the adaptive controller which lets the closed-loop system be still stable in the event of actuator fault. $u_{\sigma}\left(t, t_{\sigma}\right)$ is an additive bias fault; $t_{b}$ indicates the point at which the actuator loses its effectiveness, and $t_{\sigma}$ represents the time from the normal state to the first additional failure.

Assumption $1[48] b\left(t, t_{b}\right)$ is an unknown time-varying efficiency factor, $b_{\min }$ and $b_{\max }$ represent the known lower and upper bound of $b\left(t, t_{b}\right)$, respectively. $u_{\sigma}\left(t, t_{\sigma}\right)$ is an unparameterizable time-varying and bounded function and it has a upper bound $u_{\max }$ such that $\left|u_{\sigma}\left(t, t_{\sigma}\right)\right| \leq u_{\max }\left(u_{\max }>0\right)$.

Remark 1 In $(2)$, if $b\left(t, t_{b}\right)=1$ and $u_{\sigma}\left(t, t_{\sigma}\right)=0$, it implies the actuator is normal. If $b\left(t, t_{b}\right) \neq 1$ and $u_{\sigma}\left(t, t_{\sigma}\right)=0$, it indicates that the actuator is in the presence of loss of effectiveness. In addition, other types of sensor faults may occur at $b\left(t, t_{b}\right)=0$ and $u_{\sigma}\left(t, t_{\sigma}\right) \neq 0$. As is indicated in Assumption 1 , we consider the slow-varying fault whose range of variation is not over 1 . It is also worth noting that the additive fault $u_{\sigma}\left(t, t_{\sigma}\right)$ cannot be arbitrarily large and is bounded at least by the physical limitation of actuators. Meanwhile, the occurrence time is unpredictable for time-varying actuator faults, which may deteriorate system performances and bring certain difficulties to FTC design.

Remark 2 It is noted that there exist no any constraints on the nonlinear term $f_{i}\left(\bar{x}_{i}\right)$ in this paper, which means that the traditional boundary conditions are not required for the considered nonlinearity. Hence, more generalized nonlinear systems are considered in this work.

Definition 1 [44] Give a nonlinear system described by

$$
\dot{x}(t)=g(x(t)), t>t_{0}
$$

in which $g(\cdot)$ is continuous, $x(0)=x_{0}$, and $x \in R^{n}$ is the state vector. If by constructing the Lyapunov function (3) is globally asymptotically stable with all solutions $x\left(t, x_{0}\right)$ of $(3)$ converging in finite time, for $t \geq t_{\Phi}$, there is finite convergence time $T_{\Phi}\left(x_{0}\right)$ that satisfies $T \leq T_{\Phi}\left(x_{0}\right)$. Thus the origin of $(3)$ is globally finite-time stable.

Definition 2 [44] In convergence time, if there is a certain settling time that satisfies $T \leq T_{\Phi}$ and the system (3) is globally finite-time stable, the system (3) is fixed-time stable (FTS).

Lemma 1 [51, 52] Consider the system (3), for some scalars $\varrho_{1}>0, \varrho_{2}>0,0<p<1, q>1$ and $\nu>0$ and $0<\varpi<1$ and if there exists a positive smooth function $V(x)$ such that

$$
\dot{V}(x) \leq-\varrho_{1} V^{p}(x)-\varrho_{2} V^{q}(x)+\nu
$$

then origin of (3) is FTS. Furthermore, for any solution of (3), if there exists the residual set : $\left\{\lim _{t \rightarrow T_{\Phi}} x \mid\right.$ $V(x)\}$ satisfying the following:

$$
\left\{\lim _{t \rightarrow T_{\Phi}} x \mid V(x) \leq \min \left\{\varrho_{1}^{-\frac{1}{p}}\left(\frac{\nu}{1-\varpi}\right)^{\frac{1}{p}}, \varrho_{2}^{-\frac{1}{p}}\left(\frac{\nu}{1-\varpi}\right)^{\frac{1}{q}}\right\}\right\} .
$$

The time needed to reach the residual set is bounded as

$$
T_{\Phi} \leq \frac{1}{\varrho_{1} \varpi} \frac{1}{(1-p)}+\frac{1}{\varrho_{2} \varpi} \frac{1}{(q-1)} .
$$


Remark 3 The settling time is bounded by a priori value in fixed-time stability, which is not depended on initial condition but relying on the control parameters: $p, q, \varrho_{1}, \varrho_{2}$ and $\varpi$, which is the biggest difference from finite-time stability. Therefore, the convergence time can be preset in advance under the unknown initial value condition.

Lemma 2 [53] For any given positive constants $r_{1}, r_{2}$ and $r_{3}$, it holds that

$$
|x|^{r_{1}}|y|^{r_{2}} \leq \frac{r_{1}}{r_{1}+r_{2}} r_{3}|x|^{r_{1}+r_{2}}+\frac{r_{2}}{r_{1}+r_{2}} r_{3}^{-\frac{r_{1}}{r_{2}}}|y|^{r_{1}+r_{2}}
$$

where $x$ and $y$ are any real variables.

Lemma 3 [54] If there exist $\zeta>0, \forall z \in R$, one can get

$$
0 \leq|z|<\zeta+\frac{z^{2}}{\sqrt{z^{2}+\zeta^{2}}} .
$$

Lemma 4 [52] If $l \in[0,1]$ and there exist some real values $\varsigma_{1}, \ldots, \varsigma_{n}$, the following inequality holds

$$
\left(\varsigma_{1}+\varsigma_{2}+\ldots+\varsigma_{n}\right)^{l} \leq\left|\varsigma_{1}\right|^{l}+\ldots+\left|\varsigma_{n}\right|^{l}
$$

Lemma 5 [55] For $\varpi_{j} \geq 0, j=1, \ldots, m$, it holds that

$$
\left(\varpi_{1}+\varpi_{2}+\ldots+\varpi_{m}\right)^{2} \leq m\left(\varpi_{1}^{2}+\varpi_{2}^{2}+\ldots+\varpi_{m}^{2}\right)
$$

\subsection{Radial Basis Function Neural Networks}

System (1) contains an unknown nonlinear term, conventional backstepping method cannot satisfy the design of the controller. In this work, RBFNNs is adopted to approximate the nonlinear function. For example, on a compact set $\Omega \in R^{n}, \Theta(Z)$ is a continuous function; $\xi^{T} \Phi(Z)$ satisfies the following equality

$$
\Theta(Z)=\xi^{T} \Phi(Z)+\varsigma(Z)
$$

in which the ideal weight vector $\xi=\left[\xi_{1}, \xi_{2}, \ldots, \xi_{l}\right] \in R^{l}$; the approximation error $\varsigma(Z)$ satisfies $|\varsigma(Z)| \leq \pi$, $\pi>0 ; \Phi(Z)=\left[\Phi_{1}(Z), \ldots, \Phi_{l}(Z)\right]^{T}$ denotes the unit function vector, which composes a Gaussian function; $l>1$ is the number of RBFNNs nodes. In this paper, the basic unit of Gaussian function is selected as

$$
\Phi_{j}(Z)=\exp \left\{-\frac{\left\|z-\nu_{j}\right\|^{2}}{\eta_{j}}\right\}
$$

where $\nu_{j}=\left[\nu_{1}, \nu_{2}, \ldots, \nu_{l}\right], j=1,2, \ldots, l$ is the center in Gaussian function, and $\eta_{j}$ is the width in Gaussian function. Weight vector $\xi_{j}^{*}$ has the following form

$$
\xi_{j}^{*}=\arg \min _{\xi_{j} \in R^{l}}\left\{\left[\sup _{x \in \Omega}\left|\Theta_{j}\left(\bar{Z}_{j}\right)-\xi_{j}^{T} \Phi_{j}\left(Z_{j}\right)\right|\right]\right\}
$$

where $\xi_{j}^{*}=\hat{\xi}_{j}+\tilde{\xi}_{j}$. Before the controller design, the following assumptions are given.

Assumption 2 There exists a sufficiently large compact set $\Omega$ but not infinity, which can cover the system operation field.

Remark 4 In the practical application, the value of the state variable cannot be infinite, and we also have strict requirements for the approximation of nonlinear functions, which cannot be certainly approximated on the basis of infinity. Therefore, it is necessary for Assumption 2 to set up, and all subsequent work will be discussed based on this domain $\Omega$. 


\section{Controller Design and Stability Analysis}

\subsection{Fixed-time Controller Design}

First, a signal $y_{d}$ is introduced, whose nth derivative is bounded, available and smooth. Then, combining the backstepping method with the adaptive method, a new scheme will be proposed. The classic form of backstepping is as follown below

$$
\begin{aligned}
& z_{1}=y-y_{d} \\
& z_{j}=x_{j}-\varphi_{j-1}\left(\bar{z}_{j-1}\right), j=2, \ldots, m
\end{aligned}
$$

where $\varphi_{j}\left(\bar{z}_{j}\right)$ is a virtual control law to be designed and $\bar{z}_{j}$ denotes $\left[z_{1}, z_{2}, \ldots, z_{j}\right]$. By using the backstepping approach, one can generate a total control law that forces subsystems to follow the required trajectory for each segment. The details are described as follows

Step 1: Taking the time derivative of $z_{1}$ with consideration of the system (1), then we get the following results

$$
\begin{aligned}
\dot{z}_{1} & =x_{2}+f_{1}\left(x_{1}\right)-\dot{y}_{d} \\
& =z_{2}+\varphi_{1}+f_{1}\left(x_{1}\right)-\dot{y}_{d} .
\end{aligned}
$$

A Lyapunov function is constructed as follows

$$
V_{1}=\frac{1}{2} z_{1}^{2}+\frac{1}{2 b_{1}} \tilde{\theta}_{1}^{2}
$$

where $\tilde{\theta}_{1}=\theta_{1}-\hat{\theta}_{1}$ is the estimation error in which $\hat{\theta}_{1}$ is the estimation of $\theta_{1}$. One has

$$
\begin{aligned}
\dot{V}_{1} & =z_{1}\left(z_{2}+\varphi_{1}+f_{1}\left(x_{1}\right)-\dot{y}_{d}\right)-\frac{1}{b_{1}} \tilde{\theta}_{1} \dot{\hat{\theta}}_{1} \\
& =z_{1}\left(z_{2}+\varphi_{1}+\Theta_{1}\left(Z_{1}\right)\right)-\frac{1}{b_{1}} \tilde{\theta}_{1} \dot{\hat{\theta}}_{1}-\frac{z_{1}^{2}}{2} .
\end{aligned}
$$

The nonlinear function $\Theta_{1}\left(Z_{1}\right)=f_{1}\left(x_{1}\right)+z_{1} / 2-\dot{y}_{d}$ cannot be available directly due to $\Theta_{1}\left(Z_{1}\right)$ being related to $f_{1}\left(x_{1}\right)$, according to RBFNNs, for $\forall \pi_{1}>0, \Theta_{1}\left(Z_{1}\right)$ is able to be approximated as

$$
\Theta_{1}\left(Z_{1}\right)=\xi_{1}^{T} \Phi_{1}\left(Z_{1}\right)+\varsigma_{1}\left(Z_{1}\right),\left|\varsigma_{1}\left(Z_{1}\right)\right| \leq \pi_{1} .
$$

Through using simple inequality scaling, one can get

$$
\begin{aligned}
z_{1} \Theta_{1} & =z_{1}\left(\xi_{1}^{T} \Phi_{1}+\varsigma_{1}\right) \\
& \leq \frac{1}{2 a_{1}^{2}} z_{1}^{2}\left\|\xi_{1}\right\|^{2} \Phi_{1}^{T} \Phi_{1}+\frac{a_{1}^{2}}{2}+\frac{z_{1}^{2}}{2}+\frac{\pi_{1}^{2}}{2} \\
& \leq \frac{1}{2 a_{1}^{2}} z_{1}^{2} \theta_{1} \Phi_{1}^{T} \Phi_{1}+\frac{a_{1}^{2}}{2}+\frac{z_{1}^{2}}{2}+\frac{\pi_{1}^{2}}{2}
\end{aligned}
$$

where $\theta_{1}=\left\|\xi_{1}\right\|^{2}, \Theta_{1}\left(Z_{1}\right)$ and $\Phi_{1}\left(Z_{1}\right)$ are abbreviated to $\Theta_{1}$ and $\Phi_{1}$, respectively. Substituting (20) into (18) yields that

$$
\dot{V}_{1} \leq z_{1} z_{2}+z_{1} \varphi_{1}+\frac{1}{2 a_{1}^{2}} z_{1}^{2} \theta_{1} \Phi_{1}^{T} \Phi_{1}+\frac{a_{1}^{2}}{2}+\frac{\pi_{1}^{2}}{2}-\frac{1}{b_{1}} \tilde{\theta}_{1} \dot{\hat{\theta}}_{1} .
$$

In order to make the subsystem stable, virtual controller is designed as

$$
\varphi_{1}=-\frac{z_{1} \tilde{\varphi}_{1}^{2}}{\sqrt{z_{1}^{2} \tilde{\varphi}_{1}^{2}+\zeta_{1}^{2}}} .
$$

By using Lemma 3 , the term $z_{1} \varphi_{1}$ in (21) can be expressed as

$$
z_{1} \varphi_{1}=-\frac{z_{1}^{2} \tilde{\varphi}_{1}^{2}}{\sqrt{z_{1}^{2} \tilde{\varphi}_{1}^{2}+\zeta_{1}^{2}}} \leq \zeta_{1}-z_{1} \tilde{\varphi}_{1}
$$

where $\tilde{\varphi}_{1}$ can be expressed as

$$
\tilde{\varphi}_{1}=c_{1,1}\left(\frac{1}{2}\right)^{p} z_{1}^{2 p-1}+c_{1,2}\left(\frac{1}{2}\right)^{q} z_{1}^{2 q-1}+\frac{1}{2 a_{1}^{2}} z_{1} \hat{\theta}_{1} \Phi_{1}^{T} \Phi_{1}
$$


and the adaptive law is

$$
\dot{\hat{\theta}}_{1}=\frac{b_{1}}{2 a_{1}^{2}} z_{1}^{2} \Phi_{1}^{T} \Phi_{1}-\bar{\psi}_{1} \hat{\theta}_{1}-\frac{c_{1}}{b_{1}} \hat{\theta}_{1}^{3}
$$

where $\zeta_{1}, c_{1,1}, c_{1,2}, c_{1}, a_{1}$ and $b_{1}$ are all positive constants. Substituting (22)-(25) into (21), one can get

$$
\begin{aligned}
\dot{V}_{1} & \leq-c_{1,1}\left(\frac{1}{2}\right)^{p} z_{1}^{2 p}-c_{1,2}\left(\frac{1}{2}\right)^{q} z_{1}^{2 q}+z_{1} z_{2}+\Delta_{1}+\frac{\bar{\psi}_{1}}{b_{1}} \tilde{\theta}_{1} \hat{\theta}_{1}+\frac{c_{1}}{b_{1}^{2}} \tilde{\theta}_{1} \hat{\theta}_{1}^{3} \\
& \leq-c_{1,1}\left(\frac{z_{1}^{2}}{2}\right)^{p}-c_{1,2}\left(\frac{z_{1}^{2}}{2}\right)^{q}+z_{1} z_{2}+\Delta_{1}+\frac{\bar{\psi}_{1}}{b_{1}} \tilde{\theta}_{1} \hat{\theta}_{1}+\frac{c_{1}}{b_{1}^{2}} \tilde{\theta}_{1} \hat{\theta}_{1}^{3}
\end{aligned}
$$

where $\Delta_{1}=\left(a_{1}^{2}+\pi_{1}^{2}+2 \zeta_{1}\right) / 2$.

Step $j(j=2, \ldots, m-1)$ : Due to $z_{j}=x_{j}-\varphi_{j-1}$, where $\varphi_{j-1}$ is a virtual control law, one obtains

$$
\begin{aligned}
\dot{z}_{j} & =\dot{x}_{j}-\dot{\varphi}_{j-1} \\
& =z_{j+1}+\varphi_{j}\left(\bar{z}_{j}\right)+f_{j}\left(\bar{x}_{j}\right)-\dot{\varphi}_{j-1} .
\end{aligned}
$$

Consider the Lyapunov function

$$
V_{j}=\frac{1}{2} z_{j}^{2}+\frac{1}{2 b_{j}} \tilde{\theta}_{j}^{2}+V_{j-1}
$$

The differential form of $V_{j}$ is

$$
\begin{aligned}
\dot{V}_{j} \leq & -\sum_{i=1}^{j-1} c_{i, 1}\left(\frac{z_{i}^{2}}{2}\right)^{p}-\sum_{i=1}^{j-1} c_{i, 2}\left(\frac{z_{i}^{2}}{2}\right)^{q}+\sum_{i=1}^{j-1} \frac{\bar{\psi}_{i}}{b_{i}} \tilde{\theta}_{i} \hat{\theta}_{i}+\sum_{i=1}^{j-1} \frac{c_{i}}{b_{i}^{2}} \tilde{\theta}_{i} \hat{\theta}_{i}^{3} \\
& +\Delta_{j-1}+z_{j} \varphi_{j}+z_{j} \Theta_{j}-\frac{z_{j}^{2}}{2}-\frac{1}{b_{j}} \tilde{\theta}_{j} \dot{\hat{\theta}}_{j}
\end{aligned}
$$

where $\Theta_{j}=f_{j}-\dot{\varphi}_{j-1}+z_{j} / 2+z_{j-1}$ denotes a function involved nonlinearity. Similarly, $\Theta_{j}$ can be decribed by

$$
\Theta_{j}=\xi_{j}^{T} \Phi_{j}+\varsigma_{j},\left|\varsigma_{j}\right| \leq \pi_{j} .
$$

Through using simple inequality scaling, one can get

$$
z_{j} \Theta_{j} \leq \frac{1}{2 a_{j}^{2}} z_{j}^{2} \theta_{j} \Phi_{j}^{T} \Phi_{j}+\frac{a_{j}^{2}}{2}+\frac{z_{j}^{2}}{2}+\frac{\pi_{j}^{2}}{2}
$$

where $\theta_{1}=\left\|\xi_{1}\right\|^{2}$. With the assistance of (23) and Lemma 3, one can get the following result

$$
\begin{gathered}
z_{j} \varphi_{j}=-\frac{z_{j}^{2} \tilde{\varphi}_{j}^{2}}{\sqrt{z_{j}^{2} \tilde{\varphi}_{j}^{2}+\zeta_{j}^{2}}} \leq \zeta_{j}-z_{j} \tilde{\varphi}_{j} \\
\tilde{\varphi}_{j}=c_{j, 1}\left(\frac{1}{2}\right)^{p} z_{j}^{2 p-1}+c_{j, 2}\left(\frac{1}{2}\right)^{q} z_{j}^{2 q-1}+\frac{1}{2 a_{j}^{2}} z_{j}^{2} \hat{\theta}_{j} \Phi_{j}^{T} \Phi_{j} .
\end{gathered}
$$

Accordingly, substituting (31) and (32) into (29), one can get the following inequalities

$$
\begin{aligned}
\dot{V}_{j} \leq & -\sum_{i=1}^{j-1} c_{i, 1}\left(\frac{z_{i}^{2}}{2}\right)^{p}-\sum_{i=1}^{j-1} c_{i, 2}\left(\frac{z_{i}^{2}}{2}\right)^{q}+\sum_{i=1}^{j-1} \frac{\bar{\psi}_{i}}{b_{i}} \tilde{\theta}_{i} \hat{\theta}_{i}+\sum_{i=1}^{j-1} \frac{c_{i}}{b_{i}^{2}} \tilde{\theta}_{i} \hat{\theta}_{i}^{3} \\
& +\zeta_{j}+\Delta_{j-1}-c_{j, 1}\left(\frac{z_{j}^{2}}{2}\right)^{p}-c_{j, 2}\left(\frac{z_{j}^{2}}{2}\right)^{q}+\frac{1}{2 a_{j}^{2}} z_{j}^{2} \tilde{\theta}_{j} \Phi_{j}^{T} \Phi_{j}+\frac{a_{j}^{2}}{2}+\frac{\pi_{j}^{2}}{2}-\frac{1}{b_{j}} \tilde{\theta}_{j} \dot{\hat{\theta}}_{j} .
\end{aligned}
$$

Similar to the form of (25), it yields

$$
\dot{\hat{\theta}}_{j}=\frac{b_{j}}{2 a_{j}^{2}} z_{j}^{2} \Phi_{j}^{T} \Phi_{j}-\bar{\psi}_{j} \hat{\theta}_{j}-\frac{c_{j}}{b_{j}} \hat{\theta}_{j}^{3}
$$

Furthermore, it follows that

$$
\dot{V}_{j} \leq-\sum_{i=1}^{j} c_{i, 1}\left(\frac{z_{i}^{2}}{2}\right)^{p}-\sum_{i=1}^{j} c_{i, 2}\left(\frac{z_{i}^{2}}{2}\right)^{q}+z_{j} z_{j+1}+\Delta_{j}+\sum_{i=1}^{j} \frac{\bar{\psi}_{i}}{b_{i}} \tilde{\theta}_{i} \hat{\theta}_{i}+\sum_{i=1}^{j} \frac{c_{i}}{b_{i}^{2}} \tilde{\theta}_{i} \hat{\theta}_{i}^{3}
$$


where $\Delta_{j}=\left(\left(2 \zeta_{j}+a_{j}^{2}+\pi_{j}^{2}\right) / 2\right)+\Delta_{j-1}$.

Step $m$ : According to the result in step $j$, the following equality is satisfied

$$
\begin{aligned}
\dot{z}_{m} & =\dot{x}_{m}-\dot{\varphi}_{m-1} \\
& =u^{F}+f_{m}-\dot{\varphi}_{m-1} \\
& =b\left(t, t_{b}\right) u_{k}+u_{\sigma}\left(t, t_{\sigma}\right)+f_{m}-\dot{\varphi}_{m-1} .
\end{aligned}
$$

Select Lyapunov function as follows

$$
V_{m}=V_{m-1}+\frac{1}{2} z_{m}^{2}+\frac{1}{2 b_{m}} \tilde{\theta}_{m}^{2} .
$$

Its derivative is given as

$$
\begin{aligned}
\dot{V}_{m} \leq & -\sum_{i=1}^{m-1} c_{i, 1}\left(\frac{z_{i}^{2}}{2}\right)^{p}-\sum_{i=1}^{m-1} c_{i, 2}\left(\frac{z_{i}^{2}}{2}\right)^{q}+\sum_{i=1}^{m-1} \frac{\bar{\psi}_{i}}{b_{i}} \tilde{\theta}_{i} \hat{\theta}_{i}+\sum_{i=1}^{m-1} \frac{c_{i}}{b_{i}^{2}} \tilde{\theta}_{i} \hat{\theta}_{i}^{3} \\
& +\Delta_{m-1}+z_{m}\left(\Theta_{m}+b\left(t, t_{b}\right) u_{k}+u_{\sigma}\left(t, t_{\sigma}\right)\right)-\frac{1}{b_{m}} \tilde{\theta}_{m} \dot{\hat{\theta}}_{m}-z_{m}^{2}
\end{aligned}
$$

where $\Theta_{m}=f_{m}-\dot{\varphi}_{m-1}+z_{m-1}+z_{m}$. Similarly, the nonlinear term $\Theta_{m}$ can be expressed as

$$
\Theta_{m}=\xi_{m}^{T} \Phi_{m}+\varsigma_{m},\left|\varsigma_{m}\right| \leq \pi_{m}
$$

and it is not difficult to arrive at

$$
z_{m} \Theta_{m} \leq \frac{1}{2 a_{m}^{2}} z_{m}^{2} \theta_{m} \Phi_{m}^{T} \Phi_{m}+\frac{a_{m}^{2}}{2}+\frac{z_{m}^{2}}{2}+\frac{\pi_{m}^{2}}{2}
$$

where $\theta_{m}=\left\|\xi_{m}\right\|^{2}$. Accordingly, combining (35), (41) with (39), it is true that

$$
\begin{aligned}
\dot{V}_{m} \leq & -\sum_{i=1}^{m-1} c_{i, 1}\left(\frac{z_{i}^{2}}{2}\right)^{p}-\sum_{i=1}^{m-1} c_{i, 2}\left(\frac{z_{i}^{2}}{2}\right)^{q}+\sum_{i=1}^{m-1} \frac{\bar{\psi}_{i}}{b_{i}} \tilde{\theta}_{i} \hat{\theta}_{i}+\sum_{i=1}^{m-1} \frac{c_{i}}{b_{i}^{2}} \tilde{\theta}_{i} \hat{\theta}_{i}^{3} \\
& +\Delta_{m-1}+\frac{1}{2 a_{m}^{2}} z_{m}^{2} \theta_{m} \Phi_{m}^{T} \Phi_{m}+\frac{a_{m}^{2}+z_{m}^{2}+\pi_{m}^{2}}{2}+z_{m} b\left(t, t_{b}\right) u_{k}+z_{m} u_{\sigma}\left(t, t_{\sigma}\right) \\
& -\frac{\tilde{\theta}_{m}}{2 a_{m}^{2}} z_{m}^{2} \Phi_{m}^{T} \Phi_{m}+\frac{\bar{\psi}_{m}}{b_{m}} \tilde{\theta}_{m} \hat{\theta}_{m}+\frac{c_{m}}{b_{m}^{2}} \tilde{\theta}_{m} \hat{\theta}_{m}^{3}-z_{m}^{2} .
\end{aligned}
$$

With the same manipulation as in (32), it follows that

$$
\begin{gathered}
u_{k}=-\frac{z_{m} \tilde{\varphi}_{m}^{2}}{\sqrt{z_{m}^{2} \tilde{\varphi}_{m}^{2}+\zeta_{m}^{2}}} \\
z_{m} b\left(t, t_{b}\right) u_{k}=-\frac{b\left(t, t_{b}\right) z_{m}^{2} \tilde{\varphi}_{m}^{2}}{b_{\min } \sqrt{z_{m}^{2} \tilde{\varphi}_{m}^{2}+\zeta_{m}^{2}}} \leq-\frac{z_{m}^{2} \tilde{\varphi}_{m}^{2}}{\sqrt{z_{m}^{2} \tilde{\varphi}_{m}^{2}+\zeta_{m}^{2}}} \leq \zeta_{m}-z_{m} \tilde{\varphi}_{m} .
\end{gathered}
$$

Then, by applying Young's inequality [56] to terms $z_{m} u_{\sigma}\left(t, t_{\sigma}\right)$, we get

$$
z_{m} u_{\sigma}\left(t, t_{\sigma}\right) \leq \frac{1}{2}\left(z_{m}^{2}+u_{\max }^{2}\right) .
$$

From (42), (44) and (45), it produces

$$
\begin{aligned}
\dot{V}_{m} \leq & -\sum_{i=1}^{m} c_{i, 1}\left(\frac{z_{i}^{2}}{2}\right)^{p}-\sum_{i=1}^{m} c_{i, 2}\left(\frac{z_{i}^{2}}{2}\right)^{q}+\sum_{i=1}^{m} \frac{\bar{\psi}_{i}}{b_{i}} \tilde{\theta}_{i} \hat{\theta}_{i}+\sum_{i=1}^{m} \frac{c_{i}}{b_{i}^{2}} \tilde{\theta}_{i} \hat{\theta}_{i}^{3} \\
& +\frac{a_{m}^{2}+\pi_{m}^{2}+2 \zeta_{m}+u_{\max }^{2}}{2}+\Delta_{m-1} \\
\leq & -\sum_{i=1}^{m} c_{i, 1}\left(\frac{z_{i}^{2}}{2}\right)^{p}-\sum_{i=1}^{m} c_{i, 2}\left(\frac{z_{i}^{2}}{2}\right)^{q}+\sum_{i=1}^{m} \frac{\bar{\psi}_{i}}{b_{i}} \tilde{\theta}_{i} \hat{\theta}_{i}+\sum_{i=1}^{m} \frac{c_{i}}{b_{i}^{2}} \tilde{\theta}_{i} \hat{\theta}_{i}^{3}+\Delta_{m}
\end{aligned}
$$

where $\Delta_{m}=\left(a_{m}^{2}+\pi_{m}^{2}+2 \zeta_{m}+u_{\max }^{2}\right) / 2+\Delta_{m-1}$. The proof of the controller design is completed. 
3.2 Stability Analysis

Theorem 1 For an uncertain system with actuator faults (1), the controller (43), the parameter adaptive law (35) and the virtual controller (33) are adopted where all the parameters are positive constants, which ensures the tracking errors are convergent in the fixed time with $T$ satisfying $T_{\Phi} \leq \frac{4}{\varrho_{1} \varpi}+\frac{1}{\varrho_{2} \varpi}$, the closed-loop system (1) is FTS. Thereinto, all the signals are bounded.

Proof. Consider the Lyapunov function as follows

$$
V_{m}=\frac{1}{2} z_{m}^{2}+\frac{1}{2 b_{m}} \tilde{\theta}_{m}^{2}
$$

According to the definition of $\tilde{\theta}_{i}$, one can get the following inequality

$$
\tilde{\theta}_{i} \hat{\theta}_{i}=\tilde{\theta}_{i}\left(\theta_{i}-\tilde{\theta}_{i}\right) \leq-\frac{1}{2} \tilde{\theta}_{i}^{2}+\frac{1}{2} \theta_{i}^{2}
$$

and motivated by the literature [56], just to facilitate the description of the binomial theorem $\left(\theta_{i}-\tilde{\theta}_{i}\right)^{3}$, $p=3 / 4$ and $q=2$ are used to demonstrate stability. Based on (47), (48), Lemma 5 and Lemma 6 , then the time derivative of $V_{m}$ is

$$
\begin{aligned}
\dot{V}_{m} \leq & \sum_{i=1}^{m} \frac{\bar{\psi}_{i}}{b_{i}} \tilde{\theta}_{i} \hat{\theta}_{i}+\sum_{i=1}^{m} \frac{c_{i}}{b_{i}^{2}} \tilde{\theta}_{i} \hat{\theta}_{i}^{3}-\sum_{i=1}^{m} c_{i, 1}\left(\frac{z_{i}^{2}}{2}\right)^{\frac{3}{4}}-\sum_{i=1}^{m} c_{i, 2}\left(\frac{z_{i}^{2}}{2}\right)^{2}+\Delta_{m} \\
\leq & \sum_{i=1}^{m} \frac{\bar{\psi}_{i}}{b_{i}} \tilde{\theta}_{i} \hat{\theta}_{i}+\sum_{i=1}^{m} \frac{c_{i}}{b_{i}^{2}} \tilde{\theta}_{i} \hat{\theta}_{i}^{3}-\sum_{i=1}^{m} c_{i, 1}\left(\frac{z_{i}^{2}}{2}\right)^{\frac{3}{4}}-\frac{\bar{\varrho}_{2}}{m}\left(\sum_{i=1}^{m} \frac{z_{i}^{2}}{2}\right)^{2}+\Delta_{m} \\
\leq & -\bar{\varrho}_{1}\left(\sum_{i=1}^{m} \frac{z_{i}^{2}}{2}\right)^{\frac{3}{4}}-\frac{\bar{\varrho}_{2}}{m}\left(\sum_{i=1}^{m} \frac{z_{i}^{2}}{2}\right)^{2}-\bar{\varrho}_{1}\left(\sum_{i=1}^{m} \frac{\tilde{\theta}_{i}^{2}}{2 b_{i}}\right)^{\frac{3}{4}}+\left(\sum_{i=1}^{m} \frac{\bar{\psi}_{i} \tilde{\theta}_{i}^{2}}{2 b_{i}}\right)^{\frac{3}{4}} \\
& -\sum_{i=1}^{m} \frac{\bar{\psi}_{i} \tilde{\theta}_{i}^{2}}{2 b_{i}}+\sum_{i=1}^{m} \frac{\bar{\psi}_{i} \theta_{i}^{2}}{2 b_{i}}+\sum_{i=1}^{m} \frac{c_{i}}{b_{i}^{2}} \tilde{\theta}_{i} \hat{\theta}_{i}^{3}+\Delta_{m} .
\end{aligned}
$$

where $\bar{\varrho}_{1}=\min \left(c_{1,1}, \ldots, c_{m, 1}, \bar{\psi}_{1}, \ldots, \bar{\psi}_{m}\right), \bar{\varrho}_{2}=\min \left(c_{1,2}, \ldots, c_{m, 2}\right)$.

Applying Lemma 2 to the term $\left(\frac{\bar{\psi}_{i} \tilde{\theta}_{i}^{2}}{2 b_{i}}\right)^{\gamma}$ we can obtain that

$$
\left(\frac{\bar{\psi}_{i} \tilde{\theta}_{i}^{2}}{2 b_{i}}\right)^{\gamma} \leq(1-\gamma) \gamma^{\frac{\gamma}{1-\gamma}}+\frac{\bar{\psi}_{i} \tilde{\theta}_{i}^{2}}{2 b_{i}}
$$

Combining (50) and (49), one has

$$
\dot{V}_{m} \leq-\bar{\varrho}_{1}\left(\sum_{i=1}^{m} \frac{z_{i}^{2}}{2}\right)^{\frac{3}{4}}-\frac{\bar{\varrho}_{2}}{m}\left(\sum_{i=1}^{m} \frac{z_{i}^{2}}{2}\right)^{2}-\bar{\varrho}_{1}\left(\sum_{i=1}^{m} \frac{\tilde{\theta}_{i}^{2}}{2 b_{i}}\right)^{\frac{3}{4}}+\sum_{i=1}^{m} \frac{c_{i} \tilde{\theta}_{i} \hat{\theta}_{i}^{3}}{b_{i}^{2}}+\tilde{\nu}
$$

where $\tilde{\nu}=\sum_{i=1}^{m} \frac{\bar{\psi}_{i} \theta_{i}^{2}}{b_{i}}+(1-\gamma) \gamma^{\frac{\gamma}{1-\gamma}}+\Delta_{m}$. Similar to (48), (51) can be expressed as

$$
\begin{aligned}
\dot{V}_{m} \leq & \sum_{i=1}^{m} \frac{c_{i} \theta_{i}^{3} \tilde{\theta}_{i}}{b_{i}^{2}}-\sum_{i=1}^{m} \frac{c_{i} \tilde{\theta}_{i}^{4}}{b_{i}^{2}}-\sum_{i=1}^{m} \frac{3 c_{i} \theta_{i}^{2} \tilde{\theta}_{i}^{2}}{b_{i}^{2}}+\sum_{i=1}^{m} \frac{3 c_{i} \theta_{i} \tilde{\theta}_{i}^{3}}{b_{i}^{2}} \\
& -\bar{\varrho}_{1}\left(\sum_{i=1}^{m} \frac{z_{i}^{2}}{2}\right)^{\frac{3}{4}}-\frac{\bar{\varrho}_{2}}{m}\left(\sum_{i=1}^{m} \frac{z_{i}^{2}}{2}\right)^{2}-\bar{\varrho}_{1}\left(\sum_{i=1}^{m} \frac{\tilde{\theta}_{i}^{2}}{2 b_{i}}\right)^{\frac{3}{4}}+\tilde{\nu}
\end{aligned}
$$

Applying Young's inequality [56] to terms $\sum_{i=1}^{m} \frac{3 c_{i} \theta_{i} \tilde{\theta}_{i}^{3}}{b_{i}^{2}}$ and $\sum_{i=1}^{m} \frac{c_{i} \theta_{i}^{3} \tilde{\theta}_{i}}{b_{i}^{2}}$, one has

$$
\sum_{i=1}^{m} \frac{3 c_{i} \theta_{i} \tilde{\theta}_{i}^{3}}{b_{i}^{2}} \leq \sum_{i=1}^{m} \frac{3 c_{i} \theta_{i}^{4}}{4 \iota^{4} b_{i}^{2}}+\sum_{i=1}^{m} \frac{9 c_{i} \iota^{\frac{4}{3}} \tilde{\theta}_{i}^{4}}{4 b_{i}^{2}}
$$




$$
\sum_{i=1}^{m} \frac{c_{i} \theta_{i}^{3} \tilde{\theta}_{i}}{b_{i}^{2}} \leq \sum_{i=1}^{m} \frac{c_{i} \theta_{i}^{4}}{12 b_{i}^{2}}+\sum_{i=1}^{m} \frac{3 c_{i} \theta_{i}^{2} \tilde{\theta}_{i}^{2}}{b_{i}^{2}}
$$

Combining (53), (54) with (52) yields

$$
\begin{aligned}
\dot{V}_{m} \leq & -\frac{\bar{\varrho}_{2}}{m}\left(\sum_{i=1}^{m} \frac{z_{i}^{2}}{2}\right)^{2}-\bar{\varrho}_{1}\left(\sum_{i=1}^{m} \frac{z_{i}^{2}}{2}\right)^{\frac{3}{4}}-\sum_{i=1}^{m}\left(4 c_{i}-9 c_{i} \iota^{\frac{4}{3}}\right)\left(\frac{\tilde{\theta}_{i}^{2}}{2 b_{i}}\right)^{2} \\
& -\bar{\varrho}_{1}\left(\sum_{i=1}^{m} \frac{\tilde{\theta}_{i}^{2}}{2 b_{i}}\right)^{\frac{3}{4}}+\nu
\end{aligned}
$$

where $\nu=\tilde{\nu}+\sum_{i=1}^{m} \frac{3 c_{i} \theta_{i}^{4}}{4 \iota^{4} b_{i}^{2}}+\sum_{i=1}^{m} \frac{c_{i} \theta_{i}^{4}}{12 b_{i}^{2}}$. Moreover, (55) can be rewritten as

$$
\dot{V}_{m} \leq-\bar{\varrho}_{1}\left(\sum_{i=1}^{m} \frac{z_{i}^{2}}{2}\right)^{\frac{3}{4}}-\bar{\varrho}_{1}\left(\sum_{i=1}^{m} \frac{\tilde{\theta}_{i}^{2}}{2 b_{i}}\right)^{\frac{3}{4}}-\hat{\varrho}_{2}\left(\sum_{i=1}^{m} \frac{\tilde{\theta}_{i}^{2}}{2 b_{i}}\right)^{2}-\hat{\varrho}_{2}\left(\sum_{i=1}^{m} \frac{z_{i}^{2}}{2}\right)^{2}+\nu
$$

where $\hat{\varrho}_{2}=\min \left(\left(4-9 \iota^{\frac{4}{3}}\right) c_{i}, \frac{\bar{\rho}_{2}}{m}\right)$, we can get

$$
\begin{aligned}
\dot{V}_{m} \leq & -\bar{\varrho}_{1}\left(\left(\sum_{i=1}^{m} \frac{z_{i}^{2}}{2}\right)^{\frac{3}{4}}+\left(\sum_{i=1}^{m} \frac{\tilde{\theta}_{i}^{2}}{2 b_{i}}\right)^{\frac{3}{4}}\right) \\
& -\hat{\varrho}_{2}\left(\left(\sum_{i=1}^{m} \frac{z_{i}^{2}}{2}\right)^{2}+\left(\sum_{i=1}^{m} \frac{\tilde{\theta}_{i}^{2}}{2 b_{i}}\right)^{2}\right)+\nu .
\end{aligned}
$$

Applying Lemma 4 and Lemma 5 to $\dot{V}_{m}$, one can get

$$
\dot{V}_{m} \leq-\varrho_{1} V_{m}^{\frac{3}{4}}-\varrho_{2} V_{m}^{2}+\nu
$$

where $\varrho_{2}=\hat{\varrho}_{2}, \varrho_{1}=\bar{\varrho}_{1}$.

At the same time, it follows from (58) that

$$
\dot{V}_{m} \leq-\varrho_{2} V_{m}^{\frac{3}{4}}-\varpi \varrho_{1} V_{m}^{2}-(1-\varpi) \varrho_{1} V_{m}^{2}+\nu
$$

where $0<\varpi<1$. If $\nu-(1-\varpi) \varrho_{1} V_{m}^{2}<0$, by Lemma 1 , one can obtain

$$
T \leq \frac{4}{\varrho_{1} \varpi}+\frac{1}{\varrho_{2}}
$$

It also follows from (58) that

$$
\dot{V}_{m} \leq-\varpi \varrho_{2} V_{m}^{\frac{3}{4}}-\varrho_{1} V_{m}^{2}-(1-\varpi) \varrho_{2} V_{m}^{\frac{3}{4}}+\nu
$$

where $0<\varpi<1$. If $\nu-(1-\varpi) \varrho_{2} V_{m}^{2}<0$. By applying Lemma 1 , it follows

$$
T \leq \frac{1}{\varrho_{2} \varpi}+\frac{4}{\varrho_{1}}
$$

By using Lemma 1, that the system is FTS can be obtained and one can get

$$
\begin{gathered}
V(x) \leq \min \left\{\varrho_{1}^{-\frac{1}{p}}\left(\frac{\nu}{1-\varpi}\right)^{\frac{1}{p}}, \varrho_{2}^{-\frac{1}{p}}\left(\frac{\nu}{1-\varpi}\right)^{\frac{1}{q}}\right\} . \\
T_{\Phi} \leq \frac{4}{\varrho_{1} \varpi}+\frac{1}{\varrho_{2} \varpi}
\end{gathered}
$$

According to the definition of $V(x)$, it can be concluded that, for $\Xi \triangleq\left(2 \varrho_{1}^{-\frac{1}{2 p}}\left(\frac{\nu}{1-\varpi}\right)^{\frac{1}{2 p}}\right),\left|z_{j}\right| \leq \Xi$, and $\left|y-y_{d}\right| \leq \Xi$. On the basis of (58), it is not hard to derive that $\dot{V}_{m}<0$, when $V_{m}^{2} \geq \nu / \varrho_{2}$. Obviously, it can be observed that all the state variables $x_{j}$ are bounded. 
Remark 5 In the view of the proposed updating law $\tilde{\varphi}_{j}=c_{j, 1}\left(\frac{1}{2}\right)^{p} z_{j}^{2 p-1}+c_{j, 2}\left(\frac{1}{2}\right)^{q} z_{j}^{2 q-1}+\frac{1}{2 a_{j}^{2}} z_{j}^{2} \hat{\theta}_{j} \Phi_{j}^{T} \Phi_{j}$, the first term as well as the second term is used to prevent the adaptive parameter growing unboundedly and to ensure its fixed-time convergence, and the third term is used to eliminate neural network approximation error term in stability analysis.

Remark 6 To some extent, many design parameters determine the control performance. It is noted that the size of parameters $c, \theta, p$ and $q$ can tune convergence error and convergence accuracy simultaneously. Increasing $c_{j, 1}, c_{j, 2}$ and reducing $\dot{\hat{\theta}}_{j}$ will improve convergence rate and reduce the ultimate error $\left|y-y_{d}\right|$. However, the larger $\theta_{j}$ is, the poorer updating transient the system has. Similarly, the larger $c_{j, 1}, c_{j, 2}$ is, the higher control input is. $\left|y-y_{d}\right|$ is related to $\zeta_{j}$. Concretely, smaller $\zeta_{j}$ will reduce ultimate error. From Lemma $3, \zeta_{j}$ cannot be zero. The neural network approximation error will be reduced because of large neurons number $l$ resulting in affecting $\varphi_{j}$ and further affecting error. Thus, $\left|y-y_{d}\right|$ only enters into the range near the origin in fixed time due to the system functions and actuator failures being unknown.

\subsection{Simulation Results}

This section provides a numerical example to show the effectiveness of our control strategy. Consider a second order system described as follows

$$
\left\{\begin{array}{l}
\dot{x}_{1}=x_{2}+f_{1}\left(x_{1}\right) \\
\dot{x}_{2}=u^{F}+f_{2}\left(x_{1}, x_{2}\right) \\
y=x_{1}
\end{array}\right.
$$

where $f_{1}\left(x_{1}\right)=\sin \left(x_{1}\right)+\left(x_{1}^{2}\right) /\left(1+x_{1}^{2}\right), f_{2}\left(x_{1}, x_{2}\right)=x_{1}^{2} x_{2}$. An additional actuation fault $u_{\sigma}\left(t, t_{\sigma}\right)=$ $x_{2} \cos ^{2}\left(x_{1}\right)$ is set up. In addition, the time-varying coefficient is chosen as $b\left(t, t_{b}\right)=0.4+0.6 \exp (-0.2 t)$. First of all, assuming that the reference signal is $y_{d}=0.8 \sin t+\cos 0.5 t$. That the ouput signal can track the desired signal $y_{d}$ is the target of the controller. According to Theorem 1, $\tilde{\varphi}_{1}, \tilde{\varphi}_{2}$ and the adaptive law of $\hat{\theta}_{i}$ are as follows

$$
\begin{aligned}
& \tilde{\varphi}_{1}=c_{1,1}\left(\frac{1}{2}\right)^{\frac{3}{4}} z_{1}^{\frac{1}{2}}+c_{1,2}\left(\frac{1}{2}\right)^{2} z_{1}^{3}-\frac{1}{2 a_{1}^{2}} z_{1} \hat{\theta}_{1} \Phi_{1}^{T}\left(Z_{1}\right) \Phi_{1}\left(Z_{1}\right) \\
& \tilde{\varphi}_{2}=c_{2,1}\left(\frac{1}{2}\right)^{\frac{3}{4}} z_{2}^{\frac{1}{2}}+c_{2,2}\left(\frac{1}{2}\right)^{2} z_{2}^{3}-\frac{1}{2 a_{2}^{2}} z_{2} \hat{\theta}_{2} \Phi_{2}^{T}\left(Z_{2}\right) \Phi_{2}\left(Z_{2}\right) \\
& \dot{\hat{\theta}}_{1}=\frac{b_{1}}{2 a_{1}^{2}} z_{1}^{2} \Phi_{1}^{T}\left(Z_{1}\right) \Phi_{1}\left(Z_{1}\right)-\bar{\psi}_{1} \hat{\theta}_{1}-\frac{c_{1}}{b_{1}} \hat{\theta}_{1}^{3} \\
& \dot{\hat{\theta}}_{2}=\frac{b_{2}}{2 a_{2}^{2}} z_{2}^{2} \Phi_{2}^{T}\left(Z_{2}\right) \Phi_{2}\left(Z_{2}\right)-\bar{\psi}_{2} \hat{\theta}_{2}-\frac{c_{2}}{b_{2}} \hat{\theta}_{2}^{3}
\end{aligned}
$$

where $z_{1}=x_{1}-y_{d}, z_{2}=x_{2}-\varphi_{2}\left(\bar{z}_{2}\right)$. Finally, we combine RBFNNs with the designed controller, and there exist seven hidden nodes in neural networks. To accommodate actuator faults, parameters are selected as $c_{2,1}=c_{2,1}=c_{1,1}=c_{1,2}=11, a_{1}=0.1, a_{2}=0.08, c_{1}=c_{2}=0.01, b_{1}=10, b_{2}=37, \bar{\psi}_{1}=\bar{\psi}_{2}=1$, $\zeta_{1}=\zeta_{2}=0.001$. The initial values of the adaptive parameters and the state variables are chosen as $\hat{\theta}_{1}(0)=0.9, \hat{\theta}_{2}(0)=0.8$ and $x_{1}(0)=0.8, x_{2}(0)=-0.8$, respectively.

Figure 1 shows that the output can track the desired signal. In order to highlight the advantages of the proposed method, we compared the proposed method with the literature [48]. As can be seen from the Figure 1, the better tracking performance has achieved in our proposed method. Figure 2 indicates the adaptive laws of the systems are bounded. Figure 3 reveals that the tracking error is convergent. Figure 4 shows that the proposed scheme can perfectly track the given signal $y_{d}$. Figure 5 shows the trajectories of $u_{k}$ and $u^{F}$.

To better demonstrate the superiority and universality of this scheme, even if additional actuation fault and the time-varying coefficient change, the control scheme is not affected by it. They are chosen as $u_{\sigma}\left(t, t_{\sigma}\right)=\cos ^{2}\left(x_{2}\right)$ and $b\left(t, t_{b}\right)=0.8+0.1 \sin \left(x_{1} x_{2}\right)$ respectively from figure 6 to figure 9 . The remaining parameters and initial conditions remain unchanged.

One can draw a conclusion that in the fixed time, the tracking error is convergent and the system is also FTS if actuator additional fault and time-varying parameters change. 


\section{Conclusion}

A fixed-time control issue for nonlinear systems with unknown actuator failures has been addressed in this paper. A new fixed-time adaptive neural networks control scheme has been designed to cope with this problem where the unknown actuator failures are considered and the uncertain nonlinearities of the system is unknown. Compared with the existing results, the new control scheme becomes more applicable in practice. In addition, a newly adaptive fixed-time designed controller can estimate actuator faults, which can guarantee that the system is FTS. Meanwhile, all signals are bounded in the closed-loop system and the approximation of uncertain nonlinear functions is proved to be feasible. The proposed control method does not require initial conditions of the considered systems and can successfully compensate for actuator faults. Simulation results have shown that the scheme in this paper can provide faster convergence speed than finite time control. In addition, disturbance and parameterization problems are not considered in this paper, which are worth studying. For future study, we will be devoted to dealing with more complicated nonlinear functions with the help of neural network systems, which will get more conclusions about fixed-time control.

Acknowledgements This work of H. Shen was supported by the NNSFC under Grant 61873002, 62173001, 61973199 . Also, the work of J.H. Park was supported by the National Research Foundation of Korea (NRF) grant funded by the Korea government (Ministry of Science and ICT) (No. 2019R1A5A808029011).

\section{Conflict of interest}

The authors declare that they have no conflict of interest.

\section{Data Availability Statements}

Data sharing not applicable to this article as no datasets were generated or analysed during the current study.

\section{References}

1. J. Veillette: Reliable linear-quadratic state-feedback control. Automatica. 31(1), 137-143(1995)

2. F. Liao, J. Wang, G. Yang: Reliable robust flight tracking control: an LMI approach. IEEE Trans. Control Syst. Technol. 10(1), 76-89(2002)

3. G. Yang, J. Wang, Y. Soh: Reliable $H_{\infty}$ controller design for linear systems. Automatica. 37(5), $717-725(2001)$

4. W. Xie, M. Guo, B. Xu, X. Wang: Fault tolerant robust control with transients for over-actuated nonlinear systems. Nonlinear Dyn. 104(3), 2433-2450(2021)

5. F. Zhou, Y. Li, G. Liu: Robust decentralized force/position fault-tolerant control for constrained reconfigurable manipulators without torque sensing. Nonlinear Dyn. 89(2), 955-969(2017)

6. B. Mushage, J. Chedjou, K. Kyamakya: Observer-based fuzzy adaptive fault-tolerant nonlinear control for uncertain strict-feedback nonlinear systems with unknown control direction and its applications. Nonlinear Dyn,. 88(4), 2553-2575(2017)

7. T. Ma: Recursive filtering adaptive fault-tolerant tracking control for uncertain switched multivariable nonlinear systems. Int. J. Robust Nonlinear Control,.30(12), 4611-4632(2020)

8. M. Khalili, X. Zhang, Y. Cao, M. Polycarpou, T. Parisini: Distributed adaptive fault-tolerant leaderfollowing formation control of nonlinear uncertain second-order multi-agent systems. 28(15), 42874308(2018)

9. C. Wang, D. Hill, S. Ge, G. Chen: An ISS-modular approach for adaptive neural control of purefeedback systems. Automatica. 42(5), 723-731(2006)

10. J. Chang, Z. Guo, J. Cieslak, D. Henry: Robust fault accommodation strategy of the reentry vehicle: a disturbance estimate-triggered approach. Nonlinear Dyn. 103(3), 2605-2625(2021)

11. M. Du, D. Zhao, M. Yang, H. Chen: Nonlinear extended state observer-based output feedback stabilization control for uncertain nonlinear half-car active suspension systems. Nonlinear Dyn. 100(3), $2483-2503(2020)$

12. Y. Zhang, J. Jiang: Fault tolerant control system design with explicit consideration of performance degradation. IEEE Trans. Aerosp. Electron. Syst. 39(3), 838-848(2003) 
13. A. Moradvandi, M. Shahrokhi, S. A. Malek: Adaptive fuzzy decentralized control for a class of MIMO large-scale nonlinear state delay systems with unmodeled dynamics subject to unknown input saturation and infinite number of actuator failures. Inf. Sci. 475, 121-141(2019)

14. B. Xiao, Q. Hu, Y. Zhang, X. Huo: Fault-tolerant tracking control of spacecraft with attitude-only measurement under actuator failures. J. Guid. Control Dyn. 37(3), 838-849(2014)

15. M. M. Seron, J. A. De Don, J. H. Richter: Integrated sensor and actuator fault-tolerant control. Int. J. Control. 86(4), 689-708(2013)

16. W. Wang, C. Wen: Adaptive compensation for infinite number of actuator failures or faults. Automatica. 47(10), 2197-2210(2011)

17. S. Ijaz, F. Chen, M. Tariq Hamayun: A new actuator fault-tolerant control for Lipschitz nonlinear system using adaptive sliding mode control strategy. Int. J. Robust Nonlinear Control. 31(6), 23052333(2021)

18. X. Zhang, T. Parisini, M. M. Polycarpou: Adaptive fault-tolerant control of nonlinear uncertain systems: an information-based diagnostic approach. IEEE Trans. Autom. Control. 49(8), 12591274(2004)

19. Q. Shen, D. Wang, S. Zhu, E. K. Poh: Integral-type sliding mode fault-tolerant control for attitude stabilization of spacecraft. IEEE Trans. Control Syst. Technol. 23(3), 1131-1138(2015)

20. D. Zhai, L. An, J. Li, Q. Zhang: Adaptive fuzzy fault-tolerant control with guaranteed tracking performance for nonlinear strict-feedback systems. Fuzzy Sets Syst. 302, 82-100(2016)

21. D. Zhang, Z. Shen, Y. Song: Robust adaptive fault-tolerant control of nonlinear uncertain systems tracking uncertain target trajectory. Inf. Sci. 415, 446-460(2017)

22. Y. Li, Q. Wang, S. Tong: Fuzzy adaptive fault-tolerant control of fractional-order nonlinear systems. IEEE Trans. Syst. Man, Cybern. Syst. 51, 1372-1379(2021)

23. W. Yu, H. Xu, H. Feng: Robust adaptive fault-tolerant control of dynamic positioning vessel with position reference system faults using backstepping design. Int. J. Robust Nonlinear Control. 28(2), 403-415(2018)

24. S. Liu, B. Jiang, Z. Mao, S. Ding: Adaptive backstepping based fault-tolerant control for high-speed trains with actuator faults. Int. J. Control, Autom. 17(6), 1408-1420(2019)

25. W. Hao, B. Xian: Nonlinear adaptive fault-tolerant control for a quadrotor UAV based on immersion and invariance methodology. Nonlinear Dyn. 90(4), 2813-2826(2017)

26. J. Niu, F. Chen, G. Tao: Nonlinear fuzzy fault-tolerant control of hypersonic flight vehicle with parametric uncertainty and actuator fault. Nonlinear Dyn. 92(3), 1299-1315(2018)

27. H. Gao, Y. Song, C. Wen: Backstepping design of adaptive neural fault-tolerant control for MIMO nonlinear systems. IEEE Trans. Neural Netw. Learn. Syst. 28(11), 2605-2613(2017)

28. H. Zhang, Y. Cui, Y.Wang: Hybrid fuzzy adaptive fault-tolerant control for a class of uncertain nonlinear systems with unmeasured states. IEEE Trans. Fuzzy Syst. 25(5), 1041-1050(2017)

29. C. Xie, G. Yang: Decentralized adaptive fault-tolerant control for large-scale systems with external disturbances and actuator faults. Automatica. 85, 83-90(2017)

30. J. Ma, J. Park, S. Xu: Global adaptive finite-time control for uncertain nonlinear systems with actuator faults and unknown control directions. Nonlinear Dyn. 97(4), 2533-2545(2019)

31. H. Wang, W. Bai, P. Liu: Finite-time adaptive fault-tolerant control for nonlinear systems with multiple faults. IEE/CAA J. Automat. Sin. 6(6), 1417-1427(2019)

32. X. Jin, Y. He, Y. He: Finite-time robust fault-tolerant control against actuator faults and saturations. IET Control Theory Appl. 11(4), 550-556(2017)

33. Q. Xu, Z. Wang, Z. Zhen: Adaptive neural network finite time control for quadrotor UAV with unknown input saturation. Nonlinear Dyn. 98(3), 1973-1998(2019)

34. L. Wang, H. Wang, P. X. Liu: Adaptive fuzzy finite-time control of stochastic nonlinear systems with actuator faults. Nonlinear Dyn. 104(1), 523-536(2021)

35. S. Bhat, D. Bernstein: Continuous finite-time stabilization of the translational and rotational double integrators. IEEE Trans. Autom. Control. 43(5), 678-682(1998)

36. W. Wei, W. Zhang: Finite-time adaptive switched gain control for non-strict feedback nonlinear systems via nonlinear command filter. Nonlinear Dyn. 100, 3485-3496(2020)

37. Y. Cheng, H. Du, Y. He, R. Jia: Finite-time tracking control for a class of high-order nonlinear systems and its applications. Nonlinear Dyn. 76(2), 1133-1140 (2014)

38. M. Cai, Z. Xiang, J. Guo: Adaptive finite-time control for uncertain nonlinear systems with application to mechanical systems. Nonlinear Dyn. 84(2), 943-958(2016)

39. R. Sakthivel, M. Joby, C. Wang, B. Kaviarasan: Finite-time fault-tolerant control of neutral systems against actuator saturation and nonlinear actuator faults. Appl. Math. Comput. 332, 425-436(2018) 
40. P. Xu, Y. Li, S. Tong: Fuzzy adaptive finite time fault-tolerant control for multi-input and multioutput nonlinear systems with actuator faults. Int. J. Control, Autom. 17(7), 1655-1665(2019)

41. H. Ouyang, Y. Lin: Adaptive fault-tolerant control for actuator failures: a switching strategy. Automatica. 81, 87-95(2017)

42. R. Ji, J. Ma, D. Li, S. S. Ge: Finite-time adaptive output feedback control for MIMO nonlinear systems with actuator faults and saturations. IEEE Trans. Fuzzy Syst. 29(8), 2256-2270(2021)

43. X. Zhou, C. Gao, Z.-g. Li, X.-y. Ouyang, L.-b. Wu: Observer-based adaptive fuzzy finite-time prescribed performance tracking control for strict-feedback systems with input dead-zone and saturation. Nonlinear Dyn. 103(2), 1645-1661(2021)

44. A. Polyakov: Nonlinear feedback design for fixed-time stabilization of linear control systems. IEEE Trans. Autom. Control. 57(8), 2106-2110(2012)

45. Z. Chen, Y. Wang, M. Sun, Q. Sun: Convergence and stability analysis of active disturbance rejection control for first-order nonlinear dynamic systems. Trans. Inst. Meas. Control. 41(7), 2064-2076(2019)

46. G. Hu: Robust consensus tracking of a class of second-order multi-agent dynamic systems. Syst. Control Lett. 61(1), 134-142(2012)

47. J. Y. Choi, J. A. Farrell: Adaptive observer backstepping control using neural networks. IEEE Trans. Neural Netw. 12(5), 1103-1112(2001)

48. F. Wang, X. Zhang: Adaptive finite time control of nonlinear systems under time-varying actuator failures. IEEE Trans. Syst. Man, Cybern. Syst. 49(9), 1845-1852(2019)

49. M. Kale, A: Chipperfield, Stabilized mpc formulations for robust reconfigurable flight control. Control Eng. Pract. 13(6), 771-788(2005)

50. M. L. Corradini, G. Orlando: Actuator failure identification and compensation through sliding modes. IEEE Trans. Control Syst. Technol. 15(1), 184-190(2007)

51. B. Jiang, Q. Hu, M. I. Friswell: Fixed-time attitude control for rigid spacecraft with actuator saturation and faults. IEEE Trans. Control Syst. Technol. 24(5), 1892-1898(2016)

52. Z. Zhu, Y. Xia, M. Fu: Attitude stabilization of rigid spacecraft with finite-time convergence. Int. J. Robust Nonlinear Control. 21(6), 686-702(2011)

53. C. Qian, W. Lin: Non-Lipschitz continuous stabilizers for nonlinear systems with uncontrollable unstable linearization. Syst. Control Lett. 42(3), 185-200(2001)

54. C. Wang, Y. Lin: Decentralized adaptive tracking control for a class of interconnected nonlinear timevarying systems. Automatica. 54, 16-24(2015)

55. Z. Zuo, L. Tie: A new class of finite-time nonlinear consensus protocols for multi-agent systems. Int. J. Control. 87(2), 363-370(2014)

56. X. Jin: Adaptive fixed-time control for MIMO nonlinear systems with asymmetric output constraints using universal barrier functions. IEEE Trans. Autom. Control. 64(7), 3046-3053(2019) 


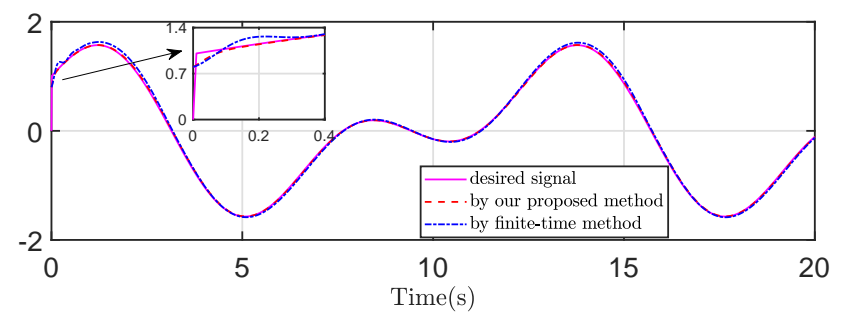

Fig. 1 Comparison of our proposed approach with finite-time method.

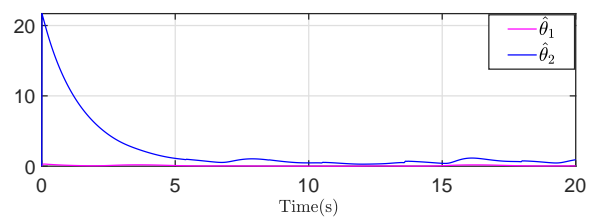

Fig. 2 Trajectories of $\hat{\theta}_{1}$ and $\hat{\theta}_{2}$

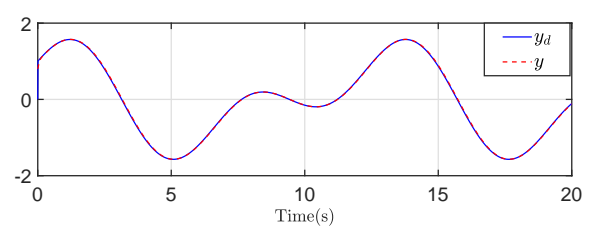

Fig. 4 Trajectories of $y$ and $y_{d}$.

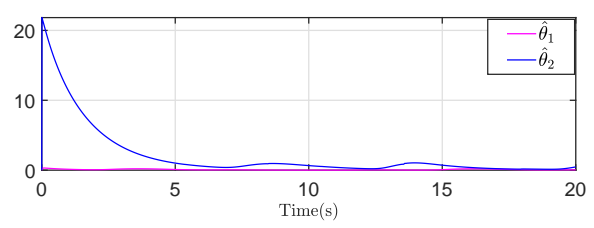

Fig. 6 Trajectories of $\hat{\theta}_{1}$ and $\hat{\theta}_{2}$

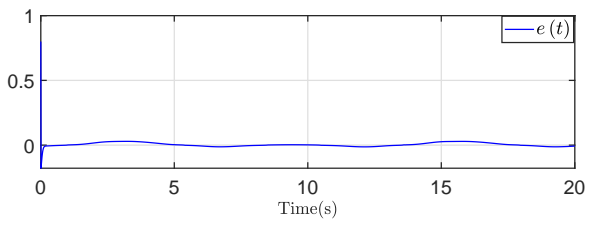

Fig. 3 Trajectory of tracking error $y-y_{d}$.

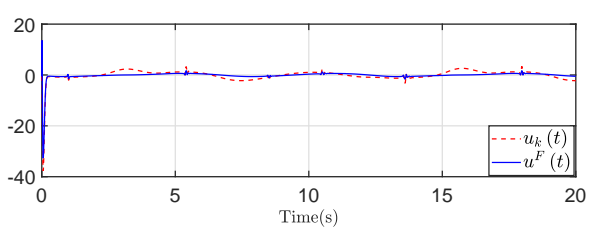

Fig. 5 Trajectories of $u_{k}$ and $u^{F}$.

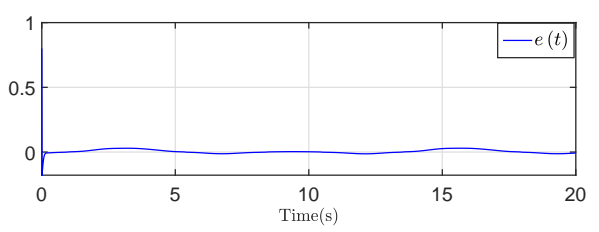

Fig. 7 Trajectory of tracking error $y-y_{d}$. 


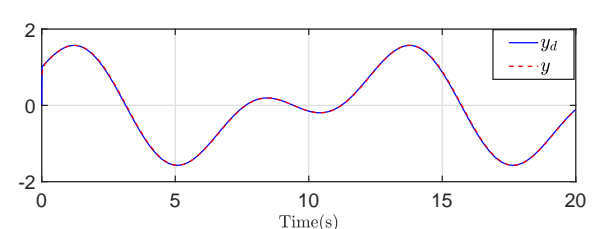

Fig. 8 Trajectories of $y$ and $y_{d}$.

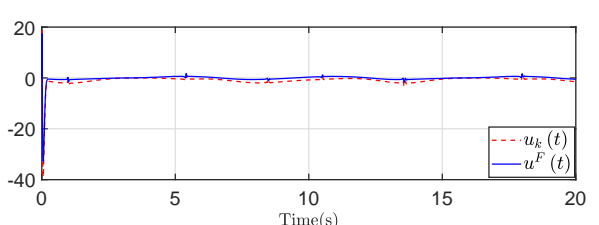

Fig. 9 Trajectory of $u_{k}$ and $u^{F}$. 